\title{
LOS EFECTOS DE LAREVEGETACIÓN EN EL MEDIO NATURAL Y SU PERCEPCIÓN POR LA POBLACIÓN VINCULADA DE CAMEROS VIEJO (SISTEMA IBÉRICO)
}

\author{
Teodoro Lasanta \\ Instituto Pirenaico de Ecología. Consejo Superior de Investigaciones Científicas \\ fm@ipe.csic.es \\ Estela Nadal-Romero \\ Departamento de Geografía y Ordenación del Territorio. Universidad de Zaragoza \\ estelanr@unizar.es \\ Pili Serrano-Muela \\ Instituto Pirenaico de Ecología. Consejo Superior de Investigaciones Científicas \\ pili@ipe.csic.es
}

\section{RESUMEN}

Se estudia la percepción que tiene la población vinculada de Cameros Viejo (Sistema Ibérico) del proceso de revegetación y sus impactos en el medio natural. Se realizaron 40 entrevistas en profundidad semi-estructuradas. Las respuestas de los entrevistados se contrastan con los resultados obtenidos en los trabajos científicos realizados en el área de estudio y en otras áreas próximas de la montaña mediterránea. Se observa que la población local tiene una percepción más realista que la población foránea de los cambios en el medio natural.

Palabras clave: campos abandonados, revegetación, conocimiento local, montaña mediterránea, España.

\section{ABSTRACT}

The perception of the population linked to Cameros Viejo (Iberian System) about the revegetation process and its impacts on the natural environment is studied. We carried out

Fecha de recepción: febrero 2014.

Fecha de aceptación: marzo 2015. 
40 depth semi-structured interviews. The interviewee' answers are corroborated with the results of scientific works carried out in the study area and in other nearby areas of the Mediterranean mountains. Moreover, it is observed that the local population shows a more realistic perception than the foreign population about the changes in the natural environment.

Keywords: abandoned fields, revegetation, local knowledge, mediterranean mountain, Spain.

\section{INTRODUCCIÓN}

Los ecosistemas de la montaña mediterránea experimentaron intensos cambios a lo largo del último siglo (Blondel, 2006; Tallefumier y Piégay, 2003). Los usos y cubiertas del suelo han cambiado como consecuencia de fuerzas socioeconómicas internas y externas a las montañas (García-Ruiz y Lasanta, 1990; Mottet et al., 2006; Serra et al., 2008). Durante siglos, se había tratado de extraer energía de la práctica totalidad del territorio, discriminando espacialmente entre las áreas dedicadas a la agricultura, al aprovechamiento ganadero y la explotación forestal, lo que dio lugar a un paisaje en mosaico, heterogéneo y de gran valor cultural (Lasanta et al., 2005; Roura-Pascual et al., 2003; Moreira et al., 2006). Sin embargo, durante el siglo XX las montañas euro-mediterráneas sufren un proceso de marginación, con elevadas tasas de emigración, abandono de tierras de cultivo, disminución de los censos ganaderos y escaso aprovechamiento de pastos y productos del bosque (MacDonald et al., 2000).

La marginación, con el abandono de la mayoría de los campos de cultivo y pastizales, incentivó procesos de sucesión secundaria que favorecieron la expansión de matorrales y bosques (Chauchard et al., 2007; Madruga-Andreu et al., 2011). De forma simultánea, la Administración incentivó la reforestación de grandes extensiones de tierras (ScarasciaMugnozza et al., 2000; Falcucci et al., 2007; Pueyo y Beguería, 2007), con fines económicos (incrementar la producción de madera y la pasta del papel) y ambientales: regular los flujos de escorrentía y evitar la erosión del suelo (Ortigosa et al., 1990; Palahi et al., 2008; Marey-Pérez y Rodríguez-Vicente, 2009). Como consecuencia de todo ello el paisaje de la montaña mediterránea se ha simplificado y homogeneizado, se ha reducido la fragmentación y muchos de sus valores culturales se están degradando (Antrop, 1997; Calvo-Iglesias et al., 2006), una tendencia que implica a la vez la pérdida de conocimientos locales sobre gestión sostenible del territorio (O’Rourke, 2006; Fernández-Giménez y Fillat, 2012 a y b).

La expansión de la vegetación tiene otros efectos negativos, como el incremento de riesgo de incendios (Santana et al., 2010, Ruiz-Mirazo y Robles, 2012), la reducción de la disponibilidad de agua en las cuencas (García-Ruiz et al., 2011), la concentración de las fuentes de sedimento en las antiguas terrazas de cultivo, que mantienen los suelos más fértiles y profundos (García-Ruiz y Lana-Renault, 2011), la pérdida de biodiversidad (Agnoletti, 2007) y problemas para la sostenibilidad económica de la montaña, tanto por la degradación de pastos (Van Auken, 2009; Komac et al., 2013), como por la pérdida de atractivo del paisaje (Sayadi et al., 2009; Morán-Ordóñez et al., 2011).

Los impactos de los cambios de uso del suelo en montaña no sólo tienen efectos a escala local, sino también a escalas regional y global, porque los ecosistemas de montaña ofrecen bienes y servicios al conjunto de la sociedad (Foley et al., 2005; Carpenter et al., 2006). El 
papel de la montaña mediterránea como proveedora de bienes y servicios es ampliamente reconocido por los científicos en las últimas décadas (de Groot et al., 2002; Fisher et al., 2009; Wallace, 2007; Costanza, 2008). Por ello, las instituciones públicas han impulsado políticas de gestión encaminadas al desarrollo sostenible, conservación de la biodiversidad y del paisaje, especialmente en territorios de alto valor natural y cultural como la montaña mediterránea (Bignal y McCracken, 2000; EEA, 2005; Strijker, 2005). Con ello las decisiones de gestión se han ido desplazado hacia organismos cada vez más alejados, sin tener en cuenta los conocimientos y la opinión de la población vinculada a un territorio (Evely et al., 2011). Se ha comprobado, sin embargo, que la aplicación de las políticas públicas es más eficaz y más rápida cuando se tiene en cuenta a la población vinculada, ya que es la encargada de ejecutarlas (Danielsen et al., 2010). Además, hay que tener en cuenta los estrechos vínculos entre ecosistemas y sistemas sociales. Ambos han evolucionado juntos, se han ido moldeando y adaptando a lo largo del tiempo hasta formar un sistema integrado de personas en la naturaleza, denominado sistema socio-ecológico o socio-ecosistema (Ostrom, 2009). Las decisiones en los sistemas sociales afectan a los ecosistemas, al igual que los cambios en los ecosistemas repercuten en el bienestar humano (Ohl et al., 2010).

El concepto población vinculada hace referencia «al conjunto de personas que tiene algún tipo de vinculación con el territorio en cuestión, ya sea porque residen allí, porque trabajan o estudian allí, o, porque, no siendo su residencia habitual, suelen pasar allí ciertos periodos de tiempo, aunque no exclusivamente por motivos vacacionales (veraneos, puentes, fines de semana...)». No se consideran vinculadas, las personas que, aunque residan temporalmente, no trabajan, o estudian, o no disponen de una segunda residencia en el territorio en cuestión (Vinuesa Angulo, 2005). Dentro de la población vinculada cabe diferenciar distintos grupos sociales, si bien una clasificación sencilla individualiza a la población local, de profunda raigambre en el lugar, constituida por agricultores y ganaderos básicamente, y al resto de usuarios o actores del territorio. En este grupo se incluyen familias que se han instalado recientemente de manera permanente y otras personas que trabajan o tienen una segunda residencia (Paniagua, 2008; López -i-Gelats et al., 2009); en adelante a este segundo grupo lo denominaremos población foránea, si bien en ocasionas formaran dos subgrupos. La convivencia en un lugar de grupos sociales diferentes permite intuir que en una misma comunidad puede haber distintas perspectivas sobre la gestión, el paisaje y el medio natural, dependiendo de su relación con el territorio, la edad, la experiencia, su nivel de estudios o su sensibilización ambiental. Conocer la percepción que tienen los grupos sociales de un espacio es de gran interés para buscar alternativas de gestión que compatibilicen la conservación de la naturaleza y el desarrollo socioeconómico (Schmitz et al., 2003 y 2007; De Aranzábal et al., 2008).

El objetivo de este trabajo es conocer la diversidad de opiniones que tiene la población vinculada de Cameros Viejo (Sistema Ibérico) sobre los principales cambios ambientales registrados desde mediados del siglo XX: dinámica de la cubierta vegetal, erosión del suelo, producción y calidad del agua, riesgo de incendios, cambios en la fauna y en el paisaje. Su percepción se relaciona con los cambios reales registrados, cuantificados a partir de cartografía de usos y cubiertas del suelo, información tomada en la Administración y bibliografía del área de estudio. Se trata, en definitiva, de averiguar qué grupos sociales tienen una percepción más próxima y más alejada de la realidad, con el fin de que los gestores del territorio tengan más información a la hora de elaborar y aplicar políticas públicas. 


\section{II. ÁREA DE ESTUDIO}

Este trabajo se ha realizado en Cameros Viejo (Sistema Ibérico, La Rioja, España). Cameros Viejo está formado por los valles del río Leza y el río Jubera (Figura 1). Ocupa una extensión de 392,67 $\mathrm{Km}^{2}$ e incluye 15 municipios que reunían un censo de 1.216 habitantes en 2012. Cameros Viejo es un área representativa de la montaña mediterránea, con altitudes comprendidas entre 600 y 1800 m s.n.m. La litología dominante incluye cuarcitas, areniscas y calizas del Mesozoico. El relieve se caracteriza por cimas alomadas y vertientes suaves, que sólo en las proximidades de los cauces incrementan su pendiente. Cuadrat y VicenteSerrano (2008) clasifican el clima como Mediterráneo de montaña. Las precipitaciones anuales oscilan entre 600 y 1.000 mm en función de la altitud y la exposición, registrándose más del $60 \%$ de la lluvia en primavera y otoño; el verano es una estación seca. La temperatura media es de $11^{\circ} \mathrm{C}$ a $600 \mathrm{~m}$ de altitud y de $6^{\circ} \mathrm{C}$ a $1.800 \mathrm{~m}$ de altitud.

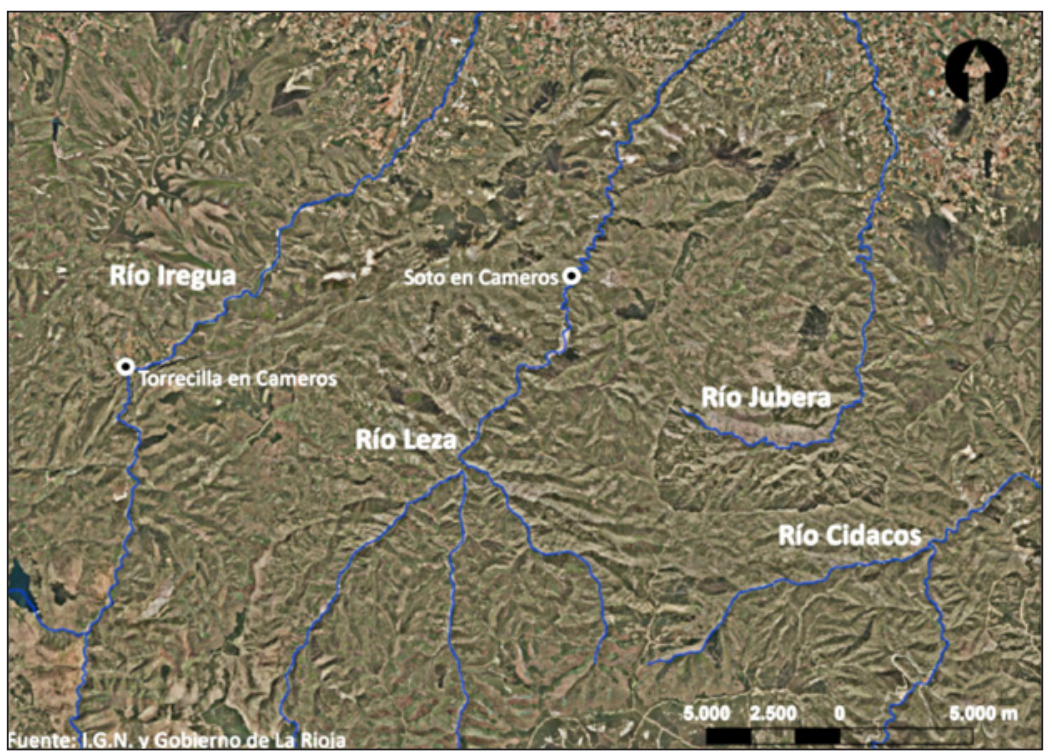

En las cimas dominan los bosques de Fagus silvatica y Quercus pyrenaica, mientras que a altitudes más bajas aparece Quercus rotundifolia sp. Balota. Algunas laderas están cubiertas por pinos, como consecuencia de su reforestación después de 1940. Los matorrales de Genista scorpius, Thymus vulgaris, Rosmarinus officinalis y Buxus sempervirens dominan en suelos calizos, mientras que Cistus laurifolius es la especie más representativa en los silíceos (Arnáez et al., 2008).

Entre los siglos XII y XVIII se deforestaron tierras para favorecer las áreas de pastoreo con las que alimentar al ganado ovino, contabilizándose un censo superior a 160.000 ovejas en la segunda mitad del siglo XVII. En el siglo XIX se roturaron muchas laderas para el cul- 
tivo de cereales con el fin de alimentar a la población local y sustituir a la economía ganadera y textil, que inició su decadencia a lo largo del siglo XIX. Durante el siglo XX, con especial intensidad en los años sesenta y setenta, la mayor parte de la población emigra, se abandona el espacio agrícola y el ganado ovino y caprino es sustituido por el vacuno (Lasanta y Errea, 2001). En 1900 la densidad era de 20,4 hab $/ \mathrm{Km}^{2}$, mientras que en 2012 era de $3,1 \mathrm{hab} / \mathrm{Km}^{2}$. El espacio agrícola ocupaba 15.974 ha a principios del siglo XX, mientras que en 2012 solo ocupaba 82 ha. En 1950 el censo de ganado ovino y caprino era de 7.012 UGM, mientras que en 2012 era de 1.299 UGM; entre las mismas fechas el vacuno pasó de 2.127 UGM a 4.486 UGM. La pérdida de población, el abandono masivo del espacio agrícola y los cambios en el sistema ganadero llevaron a un proceso muy intenso de sucesión vegetal (Lasanta y Arnáez, 2009).

\section{MÉTODOS}

\section{III.1. Entrevistas}

La valoración y percepción por la población vinculada de los cambios de cubierta vegetal y de sus impactos se obtuvo mediante entrevistas en profundidad semi-estructuradas. Durante los meses de agosto de 2011 y 2012 se realizaron 40 entrevistas semi-estructuradas entre la población vinculada (Fernández-Giménez y Fillat, 2012 a). Se seleccionaron personas de más de 40 años, con el fin de que pudieran tener una perspectiva temporal de los cambios, y de distintos sectores económicos y sensibilidad medio ambiental. Se tuvo en cuenta también su adscripción a la población local o a la foránea. Entre los componentes del primer grupo dominan agricultores y ganaderos. Entre los del segundo hay un abanico amplio de personas: funcionarios, antiguos emigrantes, nuevos residentes, propietarios de una segunda residencia... Con todos ellos se concertó una entrevista mediante una llamada de teléfono. Dejamos de realizar entrevistas cuando las adicionales ofrecían muy poca información nueva. Las entrevistas duraron de 1 a 2 horas y se llevaron a cabo en el domicilio del entrevistado, en un lugar público de su pueblo o en el campo.

En la entrevista preguntábamos sus datos personales (edad, profesión, nivel de estudios, su sensibilización medio ambiental, lugar de nacimiento y relación con el área de estudio). El segundo bloque de preguntas se dirigió a conocer su opinión sobre los cambios de cubierta vegetal en las últimas décadas (extensión y tipo) y su alcance espacial y temporal. El tercer grupo de preguntas se orientó a conocer los posibles impactos ambientales de la revegetación en la erosión del suelo, en la cantidad y calidad del agua, en el riesgo de incendios, en el paisaje y en la fauna.

Las respuestas fueron organizadas en tablas. Con el fin de establecer relaciones entre los entrevistados y el tipo de respuesta que dieron sobre las características del proceso de revegetación, se realizó un análisis de correlación canónica; este tipo de análisis multivariante facilita el estudio de las interrelaciones entre múltiples variables dependientes y múltiples variables independientes (Hair et al., 1999). Por otro lado, se tuvieron en cuenta los coeficientes beta de una regresión logística para relacionar las respuestas a los impactos ambientales con los entrevistados. Tales coeficientes nos informan de la relación entre las variables explicativas y la variable respuesta (Hair et al., 1999). Para ambos análisis se recodificó las 
respuestas en una variable ordinal que tenía valores -1 (efecto negativo), 0 (no tiene influencia) y +1 (efecto positivo). Por último, hay que recordar que valores beta mayores a 0 indican una relación positiva y valores menores a 0 una relación negativa.

\section{III.2. Información sobre la cubierta vegetal}

Para conocer la evolución de las cubiertas y usos del suelo se elaboró la cartografía de ocupación del suelo a partir de las fotografías aéreas de 1956 (escala: 1: 33000), 1981 (escala: 1: 25000) y 2006 (escala 1: 25000). Se identificaron las siguientes cubiertas y usos del suelo: espacio cultivado, bosque natural, bosque de repoblación, matorrales, pastizales, áreas erosionadas y otros usos del suelo: pueblos, ríos y roca desnuda.

A partir de bibliografía se aporta información sobre erosión del suelo en el área de estudio (Lasanta et al., 2001; Larrea et al., 2004; Arnáez et al., 2011). La evolución del número de incendios y de la superficie quemada se obtuvo del Gobierno de La Rioja; la información hace referencia al conjunto de la montaña riojana, de la que forma parte el área de estudio, para el periodo 1968-2012.

La evolución de los caudales se obtuvo de la Confederación Hidrográfica del Ebro para el periodo 1945-2006. Dado que no existen estaciones de aforo en los ríos Leza y Jubera se analizaron los caudales del Iregua y Cidacos, los dos ríos más próximos con estaciones de aforo (ver Fig. 1). En ambos casos se tomó información de las estaciones de cabecera, en concreto de Villoslada de Cameros y Yanguas, antes de que los ríos fueran regulados por embalses y antes de sustraer agua para el abastecimiento. La información sobre la calidad del agua es muy incompleta, ya que la Confederación Hidrográfica del Ebro sólo diferencia tres categorías: A1: aguas bastante buenas, que sólo necesitan un tratamiento físico y desinfección; A2: aguas que para ser potables necesitan tratamiento físico y químico normal y desinfección; A3: aguas que para ser potables necesitan tratamiento físico y químico intensivo, afino y desinfección. La información disponible cubre el periodo 1996-2012.

Por último, hay que señalar que no existe información sobre censos de especies de caza mayor. Para tener una estimación sobre su evolución se tomó información del número de batidas realizadas y de capturas de jabalí, ciervo y corzo. El Técnico del Gobierno de La Rioja, encargado del tema, nos señaló que hasta la temporada 1988-89 no se autorizaron batidas por considerar que no había superpoblación de caza mayor. A partir de dicha fecha se concedieron batidas de forma ininterrumpida hasta la actualidad. El grado de fragmentación y la diversidad del paisaje se conoció a partir de un estudio previo, en el que aplicamos índices de ecología del paisaje (Arnáez et al., 2011).

\section{RESULTADOS}

\section{IV.1. Las respuestas de los entrevistados}

En la tabla 1 se incluye el perfil de los entrevistados. De las 40 personas entrevistadas, 23 nacieron en Cameros Viejo y 17 fuera del área de estudio. La edad media era de 62 años. La distribución por edades muestra que la mayor participación corresponde a personas con edad superior a 70 años (14), como reflejo del envejecimiento de la población del área de estudio. 
No obstante, 10 de los entrevistados tenían entre 40 y 50 años. Entre ambos grupos se contó con las respuestas de 16 personas. La participación por sexos refleja el elevado índice de masculinidad del área de estudio: 10 son mujeres y 30 hombres. Dominan en la población muestreada personas con estudios primarios (24), si bien las otras dos categorías están bien representadas, especialmente las personas con estudios superiores (9). Este hecho se explica porque se tuvo especial interés en conocer la opinión de técnicos de la Administración con responsabilidad en el área de estudio (Ingenieros, Biólogos, Veterinarios,..). De ahí, que 10 de los entrevistados trabajan en el sector público, ya que a los anteriores hay que unir guardas forestales, empleados en la conservación del monte, y en ayuntamientos. En el sector primario trabajan o trabajaron antes de su jubilación 13 de los entrevistados. También colaboraron empleados de la industria (7) y del sector servicios (10).

Respecto a la relación de los entrevistados con el área de estudio cabe señalar que 13 pertenecen al grupo de la población local, siendo casi todos ellos ganaderos o agricultores; forman el Grupo A. 11 trabajan en Cameros Viejo, pero viven fuera (Grupo B). 16 no residen ni trabajan en Cameros Viejo, pero poseen una segunda residencia; algunos la casa familiar, y otros la han comprado hace más de 25 años y acuden a Cameros Viejo con mucha frecuencia (Grupo C). Por último, 16 de los entrevistados declararon que estaban preocupados por el medio ambiente, mientras que 24 señalaron que no les preocupaba especialmente.

Tabla 1

PERFIL DE LOS USUARIOS Y GESTORES ENTREVISTADOS

\begin{tabular}{|c|c|c|c|c|}
\hline \multirow{2}{*}{ Lugar de nacimiento } & \multicolumn{2}{|c|}{ Cameros Viejo } & \multicolumn{2}{|c|}{ Fuera } \\
\hline & \multicolumn{2}{|c|}{23} & \multicolumn{2}{|c|}{17} \\
\hline \multirow{2}{*}{ Edad } & $<50$ años & 51-60 años & 61-70 años & $>70$ años \\
\hline & 10 & 6 & 10 & 14 \\
\hline \multirow{2}{*}{ Sexo } & \multicolumn{2}{|c|}{ Varón } & \multicolumn{2}{|c|}{ Mujer } \\
\hline & \multicolumn{2}{|c|}{30} & \multicolumn{2}{|c|}{10} \\
\hline \multirow{2}{*}{ Estudios } & Primarios & Secundarios & Superiores & \\
\hline & 24 & 7 & 9 & \\
\hline \multirow{2}{*}{ Ocupación } & Primario & Secundario & Terciario & Sector Público \\
\hline & 13 & 7 & 10 & 10 \\
\hline \multirow{2}{*}{ Grupo social } & $\mathrm{A}$ & B & $\mathrm{C}$ & \\
\hline & 13 & 11 & 16 & \\
\hline \multirow{2}{*}{$\begin{array}{c}\text { Sensibilidad Medio } \\
\text { Ambiente }\end{array}$} & \multicolumn{2}{|c|}{ Muy sensibilizados } & \multicolumn{2}{|c|}{ Menos sensibilizados } \\
\hline & \multicolumn{2}{|c|}{16} & \multicolumn{2}{|c|}{24} \\
\hline
\end{tabular}

La tabla 2 incluye las respuestas sobre la extensión espacial y temporal del proceso de revegetación. La totalidad de los entrevistados respondieron que hay un proceso de revegetación durante las últimas décadas. El 62,5\% contesta que es un proceso intenso, el 32,5\% moderado y el $5 \%$ escaso. La mayoría de los entrevistados $(72,5 \%)$ considera que afecta a todo el territorio, mientras que el 27,5\% señala que se concentra en algunas áreas, preferentemente en los antiguos campos de cultivo. El 67,5\% opina que tanto árboles como matorrales se están expandiendo, mientras que el 32,5\% respondió que sólo los matorrales. Ninguno de los entrevistados considera que la revegetación sea un proceso exclusivo de expansión 
de árboles. Son mayoría los que opinan que la revegetación se inició al menos hace 40 años (55\%), el 22,5\% hace 30-40 años, y sólo el 22,5\% hace menos de 30 años. Nadie respondió que se inició hace menos de 10 años.

Tabla 2

RESPUESTA SOBE LA EXTENSIÓN ESPACIAL Y TEMPORAL DEL PROCESO DE REVEGETACIÓN

\begin{tabular}{|c|c|c|c|c|c|c|}
\hline $\begin{array}{c}\text { ¿Existe proceso de } \\
\text { revegetación? }\end{array}$ & \multicolumn{2}{|c|}{ Si: 40} & \multicolumn{4}{c|}{ No: 0} \\
\hline Grado & \multicolumn{2}{|c|}{ Intenso: 25} & \multicolumn{3}{c|}{ Moderado: 13} & \multicolumn{2}{c|}{ Escaso: 2} \\
\hline Localización & Generalizado: 29 & \multicolumn{4}{|c|}{ Áreas concretas: 11} \\
\hline Tipo & \multicolumn{2}{|c|}{ Matorral: 13} & \multicolumn{2}{|c|}{ Arbolado: 0} & \multicolumn{2}{c|}{ Ambos: 27} \\
\hline & $<10$ & $10-20$ & $20-30$ & $30-40$ & $40-50$ & $>50$ \\
Inicio del proceso & años: & años: & años: & años: & años: & años: \\
& 0 & 5 & 4 & 9 & 9 & 13 \\
\hline
\end{tabular}

La tabla 3 y la figura 2 hacen referencia a los resultados del análisis de correlación canónica. Sólo la primera función es significativa al 90\%, por lo que sólo comentaremos las relaciones entre las variables dependientes e independientes de la primera función. Se observa, que las cuatro variables dependientes presentan signo negativo, siendo el valor más alto el del proceso de revegetación $(-0,93)$, por la mayor unanimidad en las respuestas, mientras que los valores más bajos corresponden al tipo de revegetación $(-0,52)$ y edad de inicio $(-0,53)$. Son los agricultores y ganaderos $(-0,77)$, los incluidos en el Grupo A $(-0,77)$, los nacidos en el área de estudio $(-0,69)$ y las personas de mayor edad $(-0,37)$ los que responden que hubo un proceso intenso de revegetación, que se extiende de forma generalizada en el territorio, de bosques y matorrales y que es un proceso que se inició hace décadas. Están alejados de esta posición los que tienen mayor nivel de estudios $(0,9)$, los ambientalistas $(0,74)$, los incluidos en el Grupo B $(0,65)$, los empleados públicos $(0,52)$ y los ocupados en el sector servicios $(0,34)$. Los entrevistados incluidos en el Grupo $\mathrm{C}$ tienen menos peso, si bien su opinión se encuentra más próxima a la del Grupo B que a la del Grupo A.

Tabla 3

ANÁLISIS DE CORRELACIÓN CANÓNICA

\begin{tabular}{|c|c|c|c|}
\hline Test & Valor & Aproximación F & Significación \\
\hline Pillais & 1,066 & 1,21 & 0,09 \\
\hline Hotellings & 1,787 & 1,27 & 0,1 \\
\hline Wilks & 0,258 & 1,24 & 0,197 \\
\hline Roys & 0,512 & & \\
\hline
\end{tabular}

\begin{tabular}{|c|c|c|c|c|}
\hline Función & $\begin{array}{c}\text { Correlación } \\
\text { Canónica }\end{array}$ & $\begin{array}{c}\text { Correlación } \\
\text { canónica } \mathbf{R}^{\mathbf{2}}\end{array}$ & $\mathbf{F}$ & Significación \\
\hline 1 & 0,716 & 0,512 & 1,24 & 0,083 \\
\hline 2 & 0,558 & 0,331 & 0,83 & 0,29 \\
\hline 3 & 0,421 & 0,177 & 0,58 & 0,86 \\
\hline 4 & 0,256 & 0,065 & 0,35 & 0,9 \\
\hline
\end{tabular}




\begin{tabular}{|l|c|c|}
\hline Variables Dependientes & Función 1 & Función 2 \\
\hline Existe o no proceso de revegetación & $-0,93$ & $-0,22$ \\
\hline Localización de las áreas revegetadas & $-0,68$ & $-0,2$ \\
\hline Tipo de revegetación & $-0,52$ & 0,78 \\
\hline Inicio del proceso de revegetación & $-0,53$ & $-0,09$ \\
\hline Covariables & Función 1 & Función 2 \\
\hline Edad del entrevistado & $-0,37$ & $-0,07$ \\
\hline Sensibilización medioambiental & 0,74 & 0,12 \\
\hline Nivel de estudios & 0,9 & 0,19 \\
\hline Lugar de nacimiento & $-0,69$ & 0,22 \\
\hline Ocupación en el Primario & $-0,77$ & 0,21 \\
\hline Ocupación en el Secundarios & 0,34 & $-0,52$ \\
\hline Ocupación en el Terciario & 0,52 & 0,74 \\
\hline Grupo A & $-0,77$ & 0,17 \\
\hline Grupo B & 0,65 & 0,39 \\
\hline Grupo C & 0,14 & $-0,53$ \\
\hline
\end{tabular}

Figura 2

ANÁLISIS DE CORRELACIÓN CANÓNICA

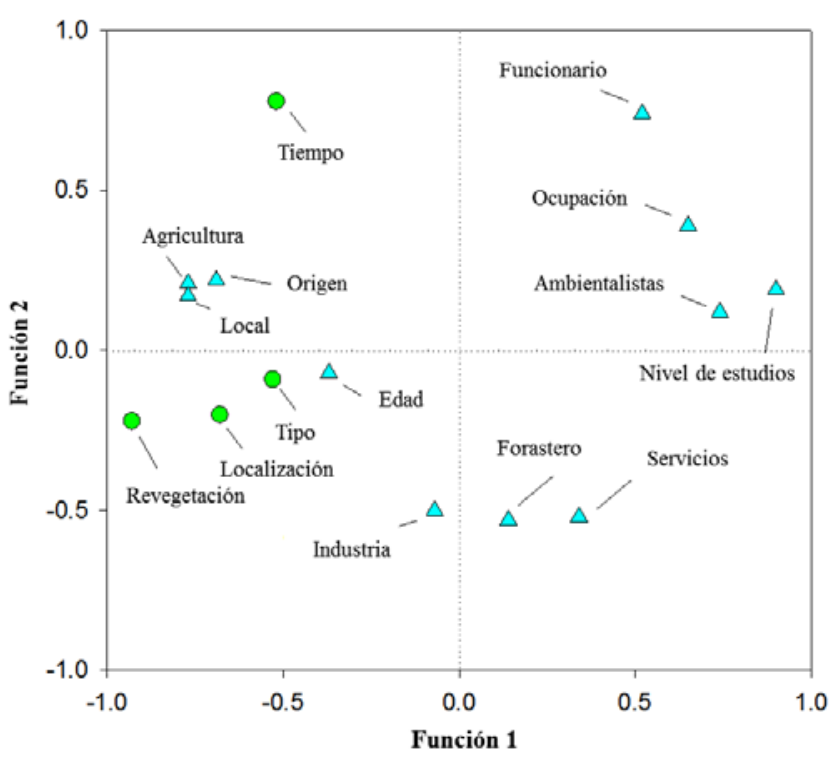

La tabla 4 muestra las opiniones de la población vinculada sobre los impactos de la revegetación en el medio natural. Las opiniones son bastante coincidentes en que el mayor cubrimiento de la vegetación disminuye la erosión del suelo (87,5\%), mientras que el 7,5\% opinó que no tiene ninguna influencia y el 5\% que hay más erosión. Tan sólo 1 entrevistado consideró que ahora hay menos riesgo de incendio, mientras que 39 opinaron que la reve- 
getación implica mayor riesgo de incendio por haber acumulación de biomasa. Mucho más divididas estuvieron las opiniones respecto al impacto en la cantidad y calidad de agua que sale de las cuencas. Respecto a la cantidad, el 62,5\% opinó que ahora los ríos llevan menos agua como consecuencia de que hay más vegetación; el 25\% manifestó lo contrario y el $12,5 \%$ que el que haya más o menos vegetación no influye en la cantidad de agua. Respecto a la calidad, el 62,5\% respondió que ahora el agua que fluye es más limpia, menos cargada de sedimentos, ya que la vegetación la filtra. El $25 \%$ opinó que la calidad del agua es ahora menor y el $12,5 \%$ que no ha cambiado en las últimas décadas. La revegetación se considera mayoritariamente $(87,5 \%)$ beneficiosa para la fauna, al aumentar los lugares donde comer y refugiarse; el $10 \%$ consideró que es negativa y el $2,5 \%$ que no tiene ninguna influencia. Las opiniones sobre el impacto de la revegetación en el paisaje estuvieron muy divididas, ya que el $65 \%$ consideró que es un proceso positivo, por resultar un paisaje más atractivo, sobre todo en primavera cuando los matorrales florecen; el 32,5\% consideró que la revegetación es negativa, ya que da lugar a un paisaje muy homogéneo y poco fragmentado. Tan sólo el 2,5\% (1 entrevistado) consideró que la revegetación no afectaba para nada al paisaje.

Tabla 4

RESPUESTAS SOBRE LOS IMPACTOS DEL PROCESO DE REVEGETACIÓN EN EL MEDIO NATURAL

\begin{tabular}{|l|c|c|c|}
\hline & \multicolumn{2}{|c|}{ Se producen } & No influye \\
\hline En la erosión del suelo & Positivos & negativos & \\
\hline En el riesgo de incendios & 35 & 2 & 3 \\
\hline En la cantidad de agua & 10 & 39 & 1 \\
\hline En la calidad del agua & 25 & 25 & 5 \\
\hline En la fauna & 35 & 10 & 5 \\
\hline En el paisaje & 26 & 4 & 1 \\
\hline
\end{tabular}

La figura 3 incluye los coeficientes beta de un modelo de regresión múltiple para explicar las respuestas a los impactos ambientales del proceso de revegetación. Las variables independientes tienen una capacidad de explicación baja, que varía entre el 56\% para la cantidad de agua y el 7\% para los incendios. Respecto al impacto de la revegetación en la erosión, las respuestas fueron positivas mayoritariamente, si bien hubo alguna opinión negativa entre los de mayor nivel de estudios, los trabajadores de los sectores secundario y terciario, el Grupo $\mathrm{C}$ y los medioambientalistas, quienes señalaron que algunos campos abancalados se estaban destruyendo. Las respuestas sobre el papel de la revegetación en el riesgo de incendios fueron bastante unánimes, lo que se refleja en la figura 3 en los valores negativos de todas las variables.

La mayor diversidad de opiniones tuvo lugar en la influencia de la revegetación en la cantidad de agua: los mayores de 65 años, con estudios primarios, que trabajan en el sector primario, pertenecientes al Grupo A y menos sensibilizados con la naturaleza respondieron que la revegetación produce menos agua, mientras que el resto de los entrevistados no tenían una opinión tan clara, señalando algunos que la revegetación implica el incremento del agua disponible en las cuencas. Las respuestas sobre la calidad del agua no establecen una divi- 
sión nítida entre los entrevistados; la mayoría manifiesta que la calidad del agua mejora si la cubierta vegetal es más extensa. No obstante, hubo personas que consideraron que empeora.

Para la mayoría la revegetación favorece a la fauna, si bien contestaron lo contrario algunos mayores de 65 años, trabajadores del secundario y terciario y menos sensibilizados con el medio ambiente. Por último, respecto a la influencia de la revegetación en el paisaje se observa que los mismos colectivos que consideraron que la revegetación es negativa para la cantidad de agua también opinaron que produce un paisaje más homogéneo y menos fragmentado; el resto consideraron que la revegetación es positiva para el paisaje.

\section{IV.2. Los cambios ambientales}

La figura 4 muestra que el espacio agrícola ocupaba una gran extensión (38\%) todavía en 1956, para descender muy rápidamente desde esa fecha: en 1981 sólo ocupaba el 1,1\% y en 2006 el 0,21\%. La superficie de las áreas erosionadas también ha disminuido del $2 \%$ en 1956 al 0,18\% en 2006. Por el contrario, los bosques naturales (frondosas y coníferas) han ido incrementando su extensión, pasando del 9,7\% en 1956 al 12,1\% en 1981 y al 17,5\% en 2006. También las repoblaciones, casi exclusivamente de coníferas, han seguido una evolución positiva: ocupaban el $0,2 \%$ en 1956 , el 6,7\% en 1981 y el 10,1\% en 2006. Los matorrales incrementaron mucho su superficie entre 1956 y 1981, pasando de ocupar el $42 \%$ al 72,4\%, para disminuir desde la última fecha hasta 2006 (51,6\%). Los pastizales se expandieron entre 1981 y 2006 (del 6,6\% al 20,2\%), mientras que en la fase anterior disminuyeron, ya que los matorrales los fueron cubriendo progresivamente. Por último, la cubierta otros usos (fundamentalmente pueblos) ha experimentado un ligero incremento pasando del $0,1 \%$ al $0,31 \%$.

Ya se ha señalado que las áreas erosionadas redujeron su superficie del $2 \%$ al $0,18 \%$ entre 1956 y 2006. Cuando el tema se analiza a escala más detallada se observa que se han borrado formas erosivas, como consecuencia del proceso de revegetación. En la actualidad las principales fuentes de sedimento se concentran en algunas laderas abancaladas y en los taludes de las pistas forestales construidas para la repoblación de coníferas (Arnáez et al., 2011). En los bancales se observan, sobre todo en laderas cóncavas y pies de vertiente, desplomes del murete externo, que dejan desprotegido y desnudo el pequeño escarpe del bancal, a partir de cuyo nicho de arranque se pueden formar pequeños surcos (Lasanta et al., 2001). En las pistas forestales se han detectado una amplia variedad de procesos de erosión que actúan con distinta intensidad, que van desde el arroyamiento difuso e incisiones a pequeños movimientos en masa, caída de tepes, pipkrakes y terracetas (Larrea et al., 2004).

La figura 5 muestra la evolución del número de incendios y de la superficie quemada en la Sierra Riojana desde 1968 hasta 2012. Se observan tres etapas: La primera abarca de 1968 a 1977. Se caracteriza por registrar pocos incendios, que además fueron muy poco activos, ya que la superficie quemada alcanzó escasa extensión. A partir de 1978 se inicia la segunda etapa que lleva hasta 1986; en ella se incrementan considerablemente el número de incendios y la superficie quemada. En esta fase la línea de la superficie quemada está siempre por encima de la del número de incendios. A partir de 1987 comienza la tercera etapa, tanto los incendios como la extensión quemada tienden a disminuir, si bien lo hace de forma mucho más clara la superficie quemada, por lo que la línea de los incendios siempre está por encima. 
Figura 3

COEFICIENTES BETA
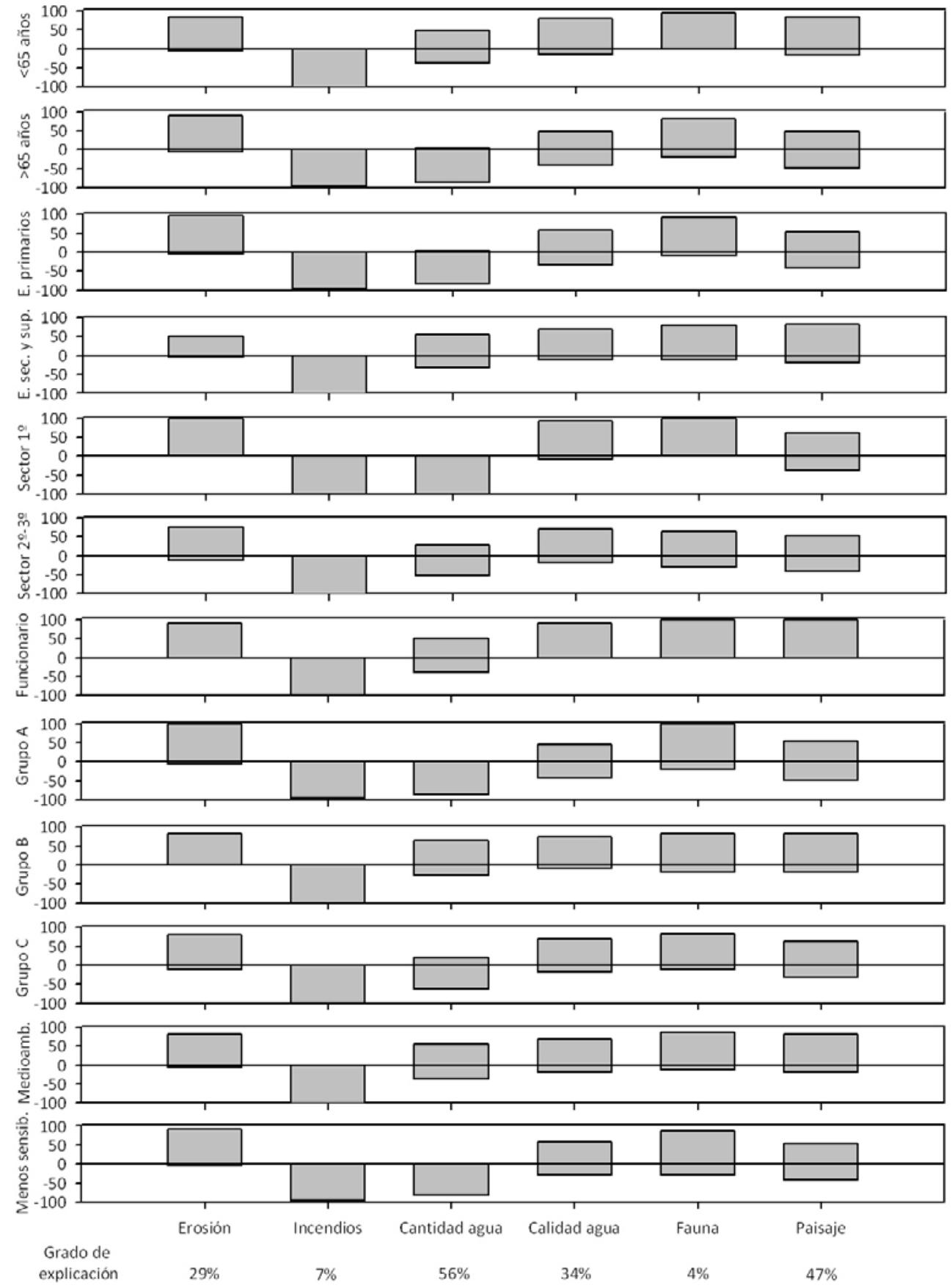


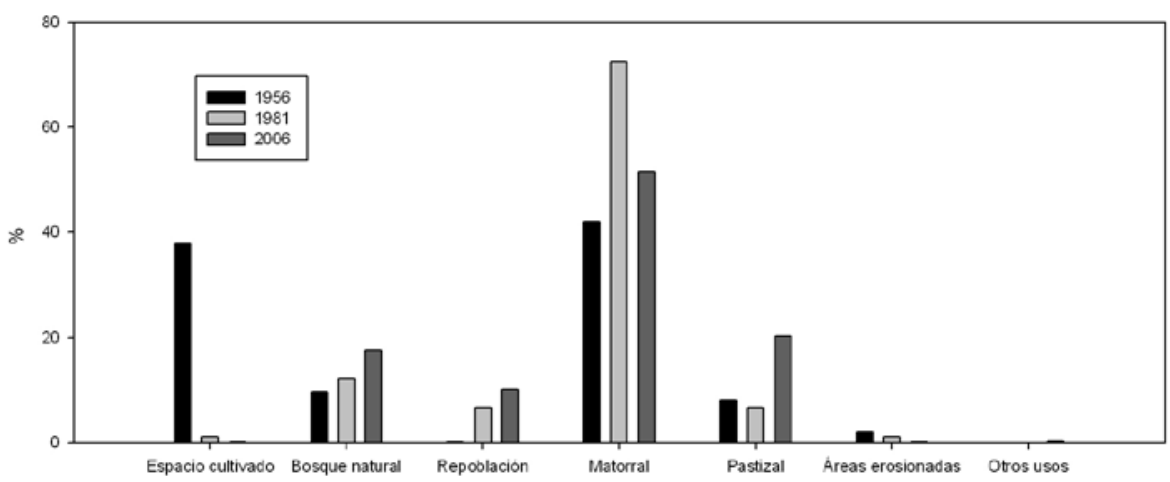

Es decir, se registran bastantes incendios pero con un impacto moderado. Dentro de esta tercera etapa podrían diferenciarse dos sub-etapas, que tendrían en 2003 el punto de inflexión. En la primera sub-etapa la separación entre ambas líneas es mayor que en la segunda, mostrando que se redujo antes la superficie quemada que el número de incendios, quizás porque todavía los ganaderos quemaban áreas de matorral para favorecer la regeneración de pastos. Desde 2003 el número de incendios se reduce muchísimo, a medida que la práctica tradicional del fuego va desapareciendo, debido a que la Administración financia el desbroce de matorrales para permitir el acceso del ganado a los pastos (Lasanta et al., 2013).

La figura 6 muestra la evolución de los caudales anuales del río Iregua en Villoslada de Cameros y del río Cidacos en Yanguas en el periodo 1945-2006. En ambos aforos se observa una gran variabilidad interanual y que la línea de tendencia desciende claramente; de hecho en Villoslada de Cameros se produce una pérdida de 3,6 $\mathrm{hm}^{3}$ por década y en Yanguas de 2,6 $\mathrm{hm}^{3}$ por década.

La información sobre la calidad del agua es poco detallada y tan sólo nos señala que desde 1996 hasta 2012 no ha variado, clasificándola todos los años como A2.

La figura 7 a muestra la evolución anual del número de batidas y las capturas de caza mayor. Se observa que el número de batidas dibuja una línea ligeramente ascendente hasta la campaña 2005-2006, en que se registró el máximo de batidas concedidas (729), mientras que en la campaña 1988-1989 fueron 439. Desde la campaña 2006-2007, el número de batidas disminuye hasta 444 en la última campaña. Por el contrario, el número de animales capturados evoluciona al alza, pasando de 603 en 1988-1989 a 5.735 en 2010-2011. Inicialmente, los jabalíes eran los animales que más se cazaban, mientras que en los últimos años jabalíes y ciervos se cazan en proporciones parecidas, mientras que los corzos se cazan mucho menos, aunque también presentan una evolución positiva (fig. 7 b).

En la tabla 5 se observa que el número de manchas ha descendido de forma acusada de 1.547 a 1.066 entre 1956 y 2001, lo que lógicamente se nota en el tamaño medio de las manchas, que ha pasado de 36 ha a 52,2 ha. El menor número de manchas ha implicado que la longitud de sus bordes también se reduzca de $4.419,5 \mathrm{~km}$ a $3.020,8 \mathrm{~km}$, al igual que la densidad de bordes. La longitud media prácticamente se mantiene en ambas fechas. El Índice de 


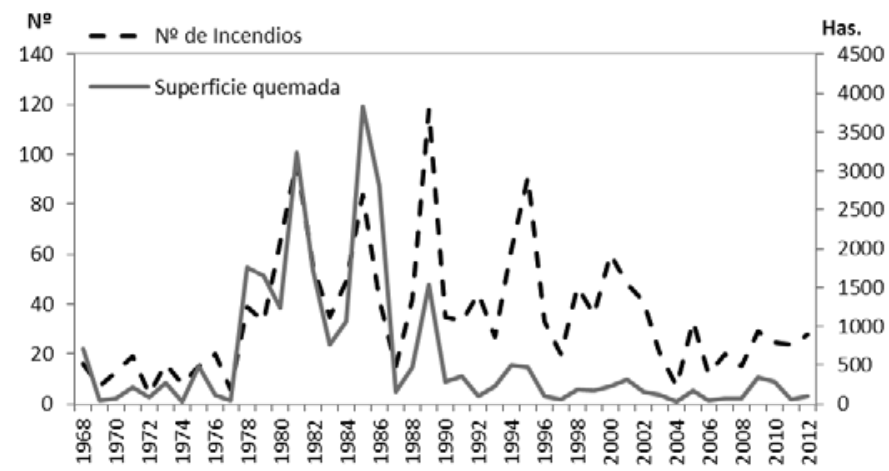

Figura 6

EVOLUCIÓN DE LOS CAUDALES ANUALES DE LOS RÍOS IREGUA Y CIDACOS
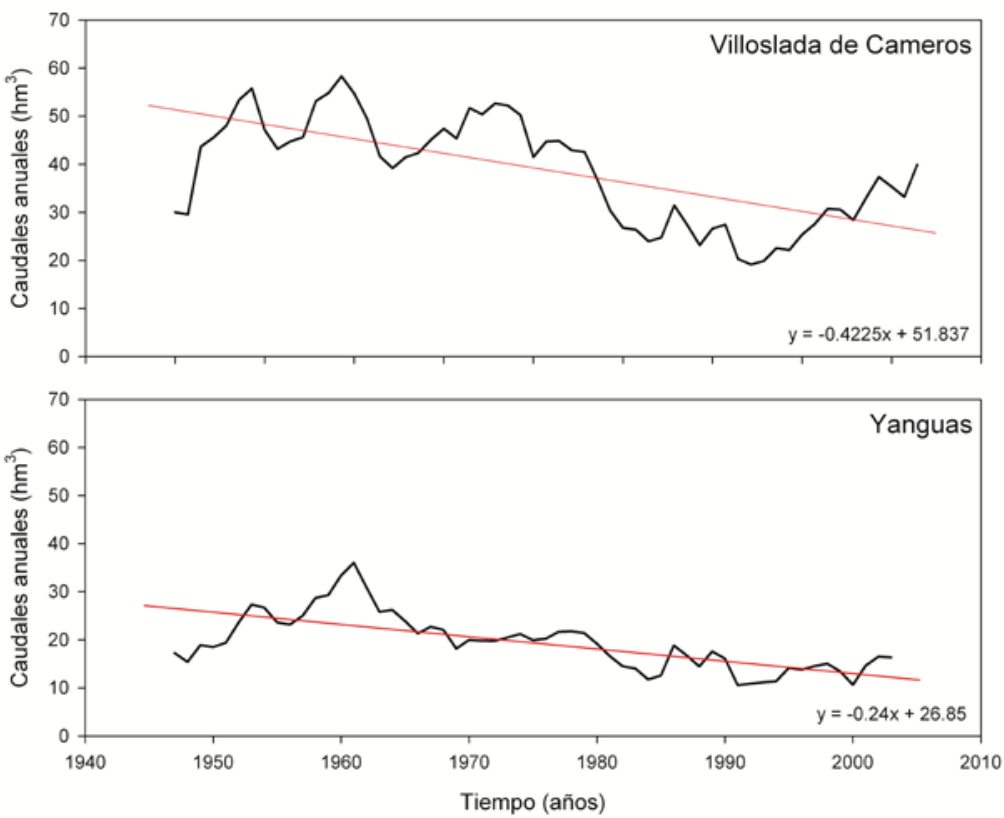

Diversidad de Shannon ha aumentado ligeramente (1,59 en 1956 y 1,658 en 2001). También el Índice de Evenness se ha incrementado un poco. Lógicamente, la dominancia ha descendido, ya que en 1956 algunas categorías cubrían elevadas proporciones del territorio. Los resultados sobre la geometría del paisaje muestran cambios mínimos, si bien el Índice Medio de Formas ha descendido, al igual que la Relación Perímetro-Área y la Dimensión Fractal. En definitiva, tenemos en 2001 un paisaje menos fragmentado y ligeramente más diverso que en 1956. 
Tabla 5

ESTRUCTURA Y DIVERSIDAD DEL PAISAJE EN CAMERO VIEJO (1956 Y 2001)

\begin{tabular}{|l|l|c|c|}
\hline \multirow{3}{*}{ ÁREA } & & 1956 & 2001 \\
\hline \multirow{4}{*}{ BORDES } & Número de manchas & 1.547 & 1.066 \\
\cline { 2 - 4 } & Tamaño de manchas (ha) & 36 & 52,2 \\
\hline \multirow{4}{*}{ DIVERSIDAD } & Longitud de bordes (Km) & $4.419,5$ & $3.020,8$ \\
\cline { 2 - 4 } & Densidad de bordes (m/ha) & 79,43 & 54,29 \\
\cline { 2 - 4 } & Media de longitud (m) & $2.856,9$ & $2.833,8$ \\
\hline \multirow{3}{*}{ FORMAS } & Shannon & 1,59 & 1,658 \\
\cline { 2 - 4 } & Evenness & 0,663 & 0,692 \\
\cline { 2 - 4 } & Dominancia & 0,808 & 0,731 \\
\cline { 2 - 4 } & Índice medio de formas & 1,476 & 1,391 \\
\cline { 2 - 4 } & Relación perímetro-área & 0,036 & 0,035 \\
\cline { 2 - 4 } & Dimensión fractal & 1,301 & 1,297 \\
\hline
\end{tabular}

Fuente: Arnáez et al. (2011).

Figura 7a

EVOLUCIÓN ANUAL DEL NÚMERO DE BATIDAS Y DE CAPTURAS DE CAZA MAYOR

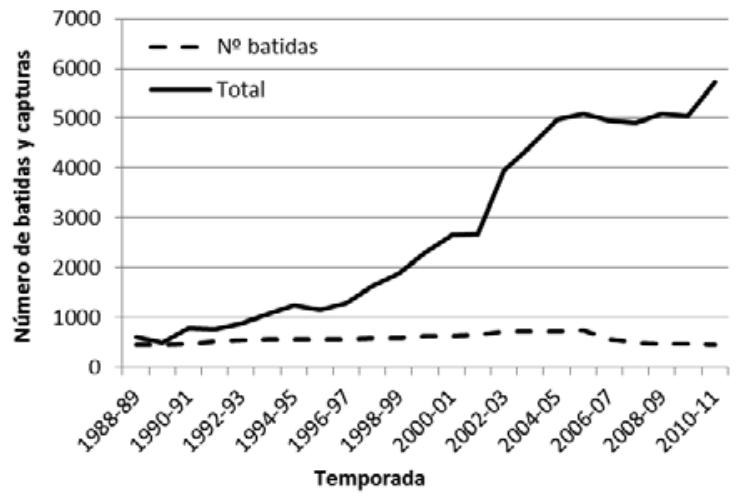

Figura $7 \mathrm{~b}$

EVOLUCIÓN DEL NÚMERO DE CAPTURAS DE JABALÍES, CIERVOS Y CORZOS

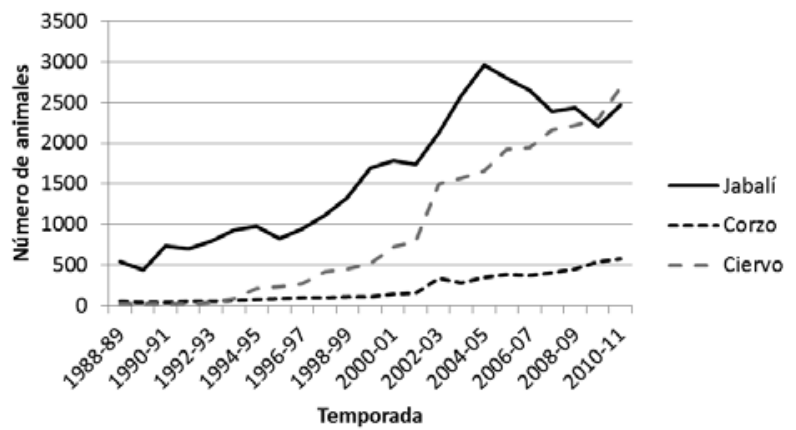




\section{DISCUSIÓN}

\section{V.1. La percepción del proceso de revegetación por la población vinculada}

Todos los entrevistados señalaron que se ha producido un proceso de revegetación en las últimas décadas. Ello se corresponde fielmente con los resultados que hemos obtenido tras realizar la cartografía de los usos y cubiertas del suelo en 1956, 1981 y 2006, donde se pone de manifiesto el abandono de campos de cultivo y la expansión de matorrales y bosques (Figura 4). Como es bien sabido, la principal consecuencia paisajística del abandono de campos de cultivo es el inicio de un proceso de sucesión secundaria que lleva a la expansión inicial de matorrales y más tarde de cubiertas forestales, proceso que ha sido estudiado en Cameros Viejo (Arnáez et al., 2009), y que también se ha observado en el Pirineo (Ubalde et al., 1999; Vicente-Serrano et al., 2005; Lasanta y Vicente-Serrano, 2007; Vila Subirós et al., 2009), y se corresponden con la tendencia más amplia de cambio de la cubierta en las tierras de cultivo abandonadas en Europa (Gellrich et al., 2007; Tasser et al., 2007; Cocca et al., 2012).

Las respuestas mayoritarias de los entrevistados indican que se han extendido tanto los matorrales como los bosques, y que ha sido un proceso generalizado, coincidiendo ambas respuestas con los resultados que refleja la cartografía. Las respuestas no fueron unánimes: las personas de mayor edad, del grupo A, nacidos en el área de estudio, con menor formación y menos sensibilizados con la conservación de la naturaleza, en definitiva la población local, tienden a opinar que es un proceso mixto (matorrales y bosques) y que ocurre por todo el territorio, mientras que entre la población foránea hay entrevistados (algunos de los más jóvenes, más sensibilizados con el medio ambiente, nivel de estudios más elevado e incluidos en los grupos B y C) que señalan que sólo se han extendido los matorrales y por áreas muy concretas. La percepción también difiere en la fecha de inicio del proceso de revegetación: la población local opina que es un proceso antiguo, de más de 40 años, mientras que la población foránea señala que es más reciente.

Este contraste de opiniones puede atribuirse, quizás, a la complejidad del proceso de sucesión vegetal, que pasa por distintas etapas: vegetación herbácea, matorral y bosque, pudiendo generar un paisaje muy diverso con áreas en distinto estadio de sucesión vegetal. El ritmo temporal y el alcance espacial de cada etapa dependen de varios factores, destacando los años transcurridos desde el cese del cultivo, la gestión, la topografía, la fertilidad y humedad del suelo, y las condiciones climáticas (Molinillo et al., 1997; González-Martínez y Bravo, 2001). Lasanta et al. (2011) estudiaron el proceso de sucesión vegetal en campos abandonados del área de estudio a partir de fotografías aéreas de 1956, 1981 y 2006. Comprobaron que los bosques se localizan en los campos abandonados antes de 1981, mientras que ocupan muy poca extensión en los abandonados después de 1981, porque no ha transcurrido el tiempo necesario para la germinación y el desarrollo de los árboles. Los matorrales se distribuyen por campos abandonados en las tres fases, poniendo de relieve la complejidad del proceso de sucesión vegetal. No obstante, en 2006 la mayor proporción de matorrales $(62,5 \%)$ se localizaba en campos abandonados entre 1956 y 1981 ; el $33 \%$ en campos abandonados antes de 1956, mientras que sólo el 4,5\% aparecía en campos abandonados en la última fase, en gran parte debido a que entre 1981 y 2006 se dejaron de cultivar ya muy 
pocas tierras (ver Fig. 4). El tema de la sucesión vegetal es aún más complejo si tenemos en cuenta que la Administración regional desbroza matorrales en Cameros Viejo para regenerar pastos y disminuir el riesgo de incendios. Lasanta et al. (2013) señalan que entre 1986 y 2012 se desbrozó el 22,2\% de la superficie de matorrales, que se transformó en pastizales.

La respuesta de la población vinculada puede estar muy condicionada por el paisaje que ellos observan más frecuentemente. Pero debe haber alguna otra razón para que la percepción de los cambios de cubierta vegetal no sea unánime; hemos señalado en los resultados que en el análisis de correlación canónica se diferencian dos grupos: la población local, por un lado, y la población foránea, por otro. Es el primer grupo el que se acerca más a la realidad de los cambios en la cubierta vegetal de Cameros Viejo. Son muchos los estudios que han documentado los profundos conocimientos que tiene la población local, sobre todo los ganaderos y agricultores, sobre los suelos, plantas, animales, agua, paisaje y otros elementos de los ecosistemas, así como sobre la gestión del territorio (Muller et al., 2007; Knapp y Fernández-Giménez, 2009; Roturier y Roue, 2009).

Entre la población foránea, sin embargo, hay varios entrevistados que tienen una percepción distorsionada de los cambios de paisaje. ¿Por qué? Resulta difícil contestar a esta pregunta y tan sólo podemos plantear hipótesis que deberíamos comprobar en estudios posteriores. Una hipótesis podría ser que su mayor preocupación por el medio ambiente les llevara a considerar que el proceso de revegetación ha sido menos amplio espacialmente, más tardío y menos diverso que lo que ellos consideran necesario. Otra apuntaría a que la población foránea tiende a distorsionar la realidad paisajística, como consecuencia de su escasa o nula dependencia económica del medio físico, algo que no ocurre con los agricultores y ganaderos que integran mayoritariamente la población local. Una tercera hipótesis plantearía que la población foránea interpreta el paisaje con algunos prejuicios, por disponer de mayor información a escala global que la población local; así -por ejemplo- el hecho de que a escala global los procesos de deforestación dominen a los de revegetación (Chowdhury y Moran, 2012), les llevaría a percibir que la expansión de la vegetación en Cameros Viejo ha sido muy modesta, porque consideran que debería ser mayor para compensar las pérdidas en otras partes del Mundo. Soliva (2007) señala que en cada sistema social se constituyen grupos de individuos con diferente percepción o visión del socio-ecosistema, en función de sus gustos o los objetivos que pretenden alcanzar.

\section{V.2. La percepción de los impactos ambientales del proceso de revegetación}

Los entrevistados opinaron mayoritariamente que la revegetación implica un descenso de las tasas de erosión en las laderas y la concentración de las principales fuentes de sedimento en algunos bancales. Ello coincide con lo señalado por la bibliografía para el área de estudio y para otras montañas mediterráneas. Se ha comprobado que el abandono de tierras implica, generalmente, un descenso en la producción de sedimentos con respecto a la fase de cultivo. La disminución de las tasas de erosión tiene que ver directamente con el aumento de la cubierta vegetal y con el descenso de la escorrentía, como consecuencia del incremento de la materia orgánica en el suelo y por una mayor capacidad de infiltración del agua y de resistencia del suelo al splash (Kosmas et al., 2000). En la Estación Experimental «Valle de Aísa» (Pirineo Central español), donde se reproducen los usos del suelo tradicionales de la 
agricultura mediterránea de montaña (cereal, barbecho y campos de agricultura nómada) y los resultantes tras el abandono de los cultivos y una parcela Testigo cubierta al $100 \%$ por Genista scorpius y otros matorrales, se ha comprobado que la parcela Testigo aporta los valores más bajos de exportación de sedimentos, y que las parcelas abandonadas presentan tasas más bajas de erosión que las parcelas cultivadas (Lasanta et al., 2010; Nadal-Romero et al., 2011). Por otro lado, en la misma estación experimental se comprobó que las mayores pérdidas de nutrientes (nitratos, fósforo, potasio, magnesio, carbonatos y calcio, entre otros) se registraron en las parcelas agrícolas, mientras que las más bajas en la parcela Testigo; las pérdidas también fueron bajas en las parcelas abandonadas, disminuyendo a medida que los matorrales ocupaban más superficie (García-Ruiz et al., 1995). Según García-Ruiz et al . (2010) el abandono de tierras de cultivo a lo largo del siglo XX es probablemente el principal factor de la reducción de aporte de sedimento hacia los embalses de la cuenca mediterránea. En definitiva, la expansión de la vegetación implica el descenso de las tasas de erosión del suelo y que el agua salga menos cargada de sedimentos y nutrientes, por lo que mejora su calidad. No obstante, en laderas cultivadas con terrazas, el abandono puede llevar a incrementos de las tasas de erosión respecto a la fase de cultivo, como consecuencia de la caída de los muretes externos de los bancales (García-Ruiz et al., 1988; Cammeraat et al., 2005; Koulouri y Giurga, 2007; Lesschen et al., 2008).

La casi totalidad de los entrevistados opinaron que ahora hay menos riesgo de incendio. La evolución del número de incendios y de la superficie quemada en el área de estudio es bastante coincidente con lo ocurrido en España y en el resto de países euro-mediterráneos (Pausas y Keeley, 2009). El incremento durante los años setenta y ochenta encuentra su explicación en el éxodo rural masivo en las décadas precedentes, lo que supuso el abandono de tierras, la disminución del pastoreo y la menor extracción de leñas y broza como fuente de energía. La consecuencia fue un aumento de la biomasa y de la combustibilidad, que llevó al incremento del número de incendios y de la superficie quemada (Moreira et al., 2001; Martínez et al., 2009). Desde finales de los años ochenta la superficie quemada ha disminuido tanto en el área de estudio como en el conjunto de España, lo que se explica por la mejora de los medios de detección temprana de los focos de incendio y de extinción, si bien el número de incendios solo muy recientemente se ha reducido (Mataix-Solera y Cerdà, 2009). El distinto ritmo de caída de la superficie quemada y del número de incendios hace pensar que es necesario llevar a cabo una mejor política de prevención para evitar que el material combustible siga acumulándose, siendo una fuente potencial de grandes incendios en el futuro. En este contexto, se enmarca el plan de desbroces de matorrales del Gobierno de La Rioja, que ha logrado que apenas haya incendios desde 2003 (Lasanta et al., 2013).

Las respuestas respecto a la producción y calidad del agua estuvieron muy divididas, siendo las de la población local las más próximas a los resultados cuantitativos obtenidos en el área de estudio. Ello coincide, además, con lo que señala la bibliografía para otras áreas de la montaña mediterránea. La expansión de los matorrales y bosques implica cambios profundos en el ciclo hidrológico, habiéndose constatado la disminución de la escorrentía superficial, debido tanto a la interceptación (Llorens y Domingo, 2007) como al mayor consumo de agua por la vegetación (Gallart et al., 2002; Serrano Muela et al., 2008). En la Estación Experimental «Valle de Aísa» se comprobó que los cultivos tradicionales eran los que producían un mayor volumen de escorrentía, mientras que las parcelas abandonadas tendían a una 
rápida colonización vegetal y a un marcado descenso en la producción de agua. El matorral denso (situación que se alcanza en el Pirineo tras unos 25-30 años de abandono) registraba las menores salidas de agua (Lasanta et al., 2010). A escala de cuenca, se ha comprobado que el coeficiente de escorrentía es mucho mayor con menos cobertura de vegetación. En el Pirineo Central español se monitorizaron tres pequeñas cuencas con diferentes tipos de cubierta vegetal: bosque, campos abandonados con una cubierta dominante de matorral y pequeñas áreas de bosque, y matorral abierto con cárcavas muy activas. El coeficiente de escorrentía más alto se registró en la cuenca de matorral abierto, seguido por la cuenca de campos abandonados; el más bajó lo registró la cuenca de bosque (García-Ruiz et al., 2008).

A escala regional también se observa una disminución de los caudales. Beguería et al. (2003) estudiaron la evolución de los caudales del Pirineo Central durante los últimos 50 años, periodo que coincide con el de los mayores cambios en la cubierta vegetal. Los resultados demostraron un cambio en las relaciones anuales entre la precipitación y el caudal, con un claro descenso del caudal desde la década de los setenta, que los autores atribuyen a la expansión generalizada de matorrales y bosques como consecuencia del abandono de tierras. Gallart y Llorens (2003) comprobaron, para toda la cuenca del Ebro, un descenso notable de los aportes del Ebro en su desembocadura en el Mediterráneo, de los cuales atribuyen un tercio a la expansión de matorrales y bosques en las cabeceras fluviales, otro tercio al descenso de las precipitaciones y el último tercio al consumo de los regadíos. López-Moreno et al. (2011) tras estudiar 88 subcuencas del Ebro concluyen que el descenso de caudales registrados entre 1950 y 2006 no puede atribuirse exclusivamente a cambios en el clima, sino que existen otros factores entre los que el abandono de tierras, la disminución del pastoreo y la sucesión vegetal posterior ocupan un lugar destacado. Otros autores confirman la importancia de la cubierta vegetal en el control de los flujos hidrológicos (Bruijnzeel, 2005; Neary et al., 2009).

Diferentes estudios muestran que los ungulados silvestres disminuyeron en la Península Ibérica a lo largo de la historia, lo que llevó a la pérdida de diversidad genética. Sin embargo, desde los años cincuenta del siglo XX, aunque especialmente en las dos últimas décadas, se encuentran en pleno proceso expansivo, tanto en superficie ocupada como en número de ejemplares, debido a su recuperación natural y a traslados efectuados entre distintas áreas de montaña (Pérez et al., 2002; Bueno et al., 2010), una tendencia también observada a escala europea (Apollonio et al., 2010). Su incremento provoca daños agrícolas y colisiones con vehículos, lo que ha impulsado cambios en los criterios de gestión durante las dos últimas décadas, pasando de la conservación y fomento al control e incluso reducción de efectivos (Marco et al., 2011). Los entrevistados del Grupo A, la población de mayor edad y los menos sensibilizados con la conservación ambiental señalaron que uno de los efectos negativos de la revegetación es la expansión de la fauna silvestre, la cual ocasiona daños a la ganadería y a los huertos. En este sentido, en un estudio reciente se ha comprobado que las hozaduras de jabalí disminuyen la calidad de los pastos pirenaicos al eliminar alguna de las especies más ricas para el ganado y favorecer la expansión de otras menos nutritivas (Bueno et al., 2011).

Los entrevistados señalan mayoritariamente que la revegetación mejora el paisaje en cuanto a diversidad y calidad estética. Los análisis a partir de índices de ecología del paisaje muestran que el paisaje es más diverso en 2001 que en 1956. Ello puede ser debido a que Cameros Viejo se encontraba, entre ambas fechas, en una fase de transición de un paisaje 
fuertemente humanizado a otro de mayor naturalidad. Dicha fase es muy lenta, pasando por una primera etapa-en la que se encontraría Cameros Viejo- de mayor diversidad y menor dominancia, ya que conviven campos abandonados en distinta fase de sucesión vegetal, lo que aumenta los índices de diversidad y equitatividad, a la vez que disminuye la dominancia. No obstante, en una etapa posterior el paisaje, posiblemente, se hará más homogéneo al evolucionar las manchas de matorral hacia bosques, tal y como se ha puesto de manifiesto en el Pirineo español, donde el abandono agrícola fue anterior y el proceso de sucesión vegetal es más rápido, por un menor estrés hídrico (Lasanta et al., 2005; Roura-Pascual et al., 2005). Algunos de los entrevistados incluidos entre la población local, Grupo A, de mayor edad, agricultores y ganaderos y menos sensibilizados con el medio ambiente opinaron que ahora el paisaje era más homogéneo y con manchas más grandes (menos fragmentado), quizás porque ellos recuerdan el paisaje en plena fase de cultivo, un paisaje muy humanizado y elevado grado de diversidad. La agricultura, el pastoreo del ganado y la gestión forestal tradicional, crearon un paisaje en mosaico y altamente integrado con la heterogeneidad del territorio (Farina, 2000; Sluiter y Jong, 2007).

Los resultados obtenidos en el valle de Borau (Pirineo Central Español) muestran un descenso de la dominancia entre 1957 y 1978, su estabilización entre 1978 y 1990, y un incremento brusco a partir de esta fecha (Lasanta et al., 2005). Tales resultados parecen indicar que el abandono de las prácticas tradicionales lleva al incremento inicial de la diversidad paisajística, por la convivencia de manchas de vegetación herbácea, arbustiva y arbórea, fruto de las disparidades espacio-temporales en el proceso de colonización vegetal (Molinillo et al., 1997; Moreira et al., 2001). Sin embargo, cuando se plantean escenarios futuros se concluye que el proceso de sucesión vegetal lleva a un paisaje mucho menos fragmentado y más homogéneo, donde sólo tres usos del suelo: pastos subalpinos, bosques y prados en los fondos de valle cubrirán la totalidad del territorio (Sferi et al., 2005).

\section{CONCLUSIONES E IMPLICACIONES}

Durante siglos el hombre mantuvo en la montaña mediterránea, con una elevada inversión de energía y trabajo, un paisaje bastante humanizado y muy heterogéneo. La agricultura contribuía muy especialmente a incrementar la complejidad y diversidad del paisaje con su variada gama de cultivos, la adaptación de los usos agrícolas a los microambientes locales y el empleo de distintos modelos de campos: bancales, en pendiente, llanos y de uso esporádico o itinerante. Con el cese de los aportes de energía y mano de obra a lo largo del siglo $\mathrm{XX}$, muchas unidades de paisaje perdieron su estabilidad «artificial», activándose procesos de sucesión secundaria, lo que ha conducido progresivamente a un paisaje muy vegetado y con rasgos de naturalidad.

La población vinculada percibe dicho cambio: todos los entrevistados observan el incremento de la vegetación durante las últimas décadas. Sin embargo, hay respuestas diferentes en las características del proceso de revegetación. Por lo general, la población local, que está constituida por agricultores y ganaderos, son personas mayores, con estudios primarios y menos sensibilizados con el medio ambiente, señalan que es un proceso que afecta a todo el territorio, compuesto por matorrales y árboles, y que se inició hace más de 40 años. Por el contrario, entre la población foránea (constituida por personas más jóvenes, con estudios medios 
o superiores, que trabajan en los sectores secundario y terciario, varios de ellos son empleados públicos con cargos de responsabilidad en el área de estudio, y que declaran estar bastante sensibilizados con el medio ambiente) algunos piensan que es un proceso de expansión exclusiva de los matorrales, que colonizan áreas concretas y que se inició hace menos de 40 años.

Los entrevistados coinciden en que el proceso de revegetación implica una mejor conservación del suelo frente a la erosión y un mayor riesgo de incendios por la acumulación de biomasa. Difieren en los cambios hidrológicos que implica el proceso de revegetación; las respuestas vuelven a unir a la población local en un grupo y a la población foránea en otro. Los primeros aprecian que ahora hay menos producción de agua, pero que es un agua de mayor calidad, menos cargada de sedimentos. Entre los segundos, hay personas que opinan que ahora hay más agua que en el pasado. También se observan algunas matizaciones respecto a las consecuencias de la revegetación en la fauna, siendo la población local la que observa más claramente la progresión de la fauna silvestre. Respecto al paisaje, las respuestas son muy coincidentes en que el paisaje actual es más bonito, si bien entre la población local hay quienes recuerdan el paisaje agrícola tradicional, lo que les lleva a restar belleza al paisaje que ahora ven.

Los trabajos científicos realizados en el área de estudio y en otras áreas de la montaña mediterránea ponen de relieve que la población local tiene mejor percepción que la población foránea sobre los cambios recientes en el medio natural. Llama especialmente la atención que los empleados públicos, todos ellos con funciones de gestión, tienen la percepción más alejada de los resultados que aporta la literatura científica.

\section{AGRADECIMIENTOS}

Este trabajo se ha realizado con el apoyo financiero del proyecto: ESPAS: Escenarios de producción de agua y sedimentos a partir de cambios de vegetación y usos del suelo: efectos de la gestión activa y pasiva del territorio, financiado por el Ministerio de Economía y Competitividad (Gobierno de España) (CGL2015-65569-R). La contribución de E. NadalRomero ha sido posible gracias a un contrato Postdoctoral «Ramón y Cajal» (Ministerio de Economía y Competitividad).

\section{BIBLIOGRAFÍA}

AGNOLETTI, M. (2007): The degradation of traditional landscape in a mountain area of Tuscany durint the 19 th and $20^{\text {th }}$ centuries: implications for biodiversity and sustainable management. Forest Ecology Management, 249, 5-17.

ANTROP, M. (1997): The concept of traditional landscapes as a base for landscape evaluation and planning: The example of Flandes Region. Landscape and Urban Planning, 38, 105-117.

APOLLONIO, M., ANDERSON, R. y PUTMAN, R. (2010): European Ungulates ant their management in the 21st century. Cambridge University Press, 618 pp., Cambridge.

ARNÁEZ, J., LARREA, V. y ORTIGOSA, L. (2004): Surface runoff and soil erosion on unpaved forest roads from rainfall simulation test in Northeastern Spain. Catena, 57(1), 1-14.

ARNÁEZ, J., LASANTA, T., ERREA, M.P. y ORTIGOSA, L. (2011): Land abandonment, landscape evolution, and soil erosion in a Spanish Mediterranean mountain region. The case of Camero Viejo. Land Degradation and Development, 22, 537-550. 
ARNAEZ, J., LASANTA, T., RUIZ-FLAÑO, P. y ORTIGOSA, L. (1990): L'abandon de l'espace agricole dans la montagne submediterranéenne en Espagne (Pyrénées Centrales et Système Ibérique). Revue Géographique des Pyrénées et du Sud-Ouest, 61, 237-253.

ARNAEZ, J., ORTIGOSA, L., OSERIN, M. y LASANTA, T. (2008): Plant cover and land uses changes in the Northwest Iberiam Range (Los Cameros, La Rioja, Spain). Boletín de la Asociación de Geógrafos Españoles, 47, 401-403.

BEGUERIA, S., LOPEZ-MORENO, J.I., LORENTE, A., SEEGER, M. y GARCIA-RUIZ, J.M. (2003): Assessing the effects of climate oscillations and land-use changes on streamflow in the Central Spanish Pyrenees. Ambio, 32, 283-286.

BIGNAL, E. y McCRACKEN, D. (2000): The nature conservation value of European traditional farming systems. Environmental Review, 8, 149-171.

BLONDEL, J. (2006): The «Design» of Mediterranean landscapes: a millennial story of humans and ecological systems during the historic period. Human Ecology, 34, 713-729.

BRUIJNZEEL, L.A. (2004): Hydrological functions of tropical forest: not seeing the soil for the trees? Agriculture, Ecosystems and Environment, 109, 185-228.

BUENO, C.G., BARRIO, I.C., GARCÍA-GONZÁLEZ, R., ALADOS, C.L. y GÓMEZGARCÍA, D. (2010): In wild boar rooting affecting livestock grazing in alpine grassland? European Journal of Wildlife Management, 56, 765-770.

BUENO, C.G., BARRIO, I.C., GARCÍA-GONZÁLEZ, R., ALADOS, C.L. y GÓMEZGARCÍA, D. (2011): Assessment of wild boar rooting on ecological and pastoral values of alpine Pyrenean grassland. Pirineos, 166, 51-67.

CALVO-IGLESIAS, M.S., CRECENTE-MASEDA, R. y FRA-PALEO, U. (2006): Exploring farmer's knowledge as a source of information on past and present cultural landscape. A case study from NW Spain. Landscape Urban Planning, 78, 334-343.

CAMMERAAT, E., VAN BEEK, R. y KOOIJMAN, A. (2005): Vegetation succession and its consequences for slope stability in SE Spain. Plant and Soil, 278, 135-147.

CARPENTER, S.R., DE FRIES, R., DIETZ, T., MOONEY, H.A., POLASKY, S., REID, W.W. y SCHOLES, R.J. (2006): Millennium Ecosystem Assessment: Research needs. Science, 314, 257-258.

CHAUCHARD, S., CARCAILLET, C. y GUIBAL, F. (2007): Patterns of land-use abandonment control tree-recruitment and forest dynamics in Mediterranean mountains. Ecosystems 10, 936-948.

CHOWDHURY, R.R. y Moran, F.E. (2012): Turning the curve: A critical review of Kuznets approaches. Applied Geography, 32, 3-11.

COCCA, G., STURARO, E., GALLO, L. y RAMANZIN, M. (2012): Is the abandonment of traditional livestock farming systems the main driver of mountain landscapes change in Alpine areas. Land Use Policy, 29: 878-886.

CONSTANZA, R. (2008): Ecosystem services: multiple classification systems are needed. Biological Conservation, 141, 350-352.

CUADRAT, J.M. y VICENTE-SERRANO, S.M. (2008): Características espaciales del clima en La Rioja modelizadas a partir de Sistemas de Información Geográfica y técnicas de regresión espacial. Zubía. Monográfico, 20, 119-142. 
DANIELSEN, F., BURGESS, N.D., JENSEN, P.M. y PIRHOFER-WALZL, K. (2010): Environmental monitoring: the scale and speed of implementation varies according to the degree of people's involvement. Journal of Applied Ecology, 47, 1166-1168.

DE ARANZABAL, I., SCHMITZ, M.F., AGUILERA, P. y PINEDA, F.D. (2008): Modelling of landscape changes derived from dynamics of socio-ecological systems. A case of study in a semiarid Mediterranean landscape. Ecological Indicators, 8, 672-685.

DE GROOT, R.S., WILSON, M.A. y BOUMANS, R.M.J. (2002): A typology for the classification, description and valuation of ecosystem functions, goods and services. Ecological Economics, 41, 393-408.

EEA (2004): High Value Nature Farmland: Characteristics, trends and policy challenges. European Environmental Agency, 27 pp. Copenhagen (Denmark).

EVELY, A.C., PINARD, M., REED, M.S. y HAZEY, I. (2011): High levels of participations in conservation projects enhance learning. Conservation Letters, 4, 116-126.

FALCUCCI, A., MAIORANO, L. y BOITANI, L. (2007): Changes in land-use/land-cover patterns in Italy and their implications for biodiversity conservation. Landscape Ecology, 22, 617-631.

FARINA, A. (2000): The cultural landscape as a model for the integration of ecology and economics. BioScience, 50, 313-320.

FERNÁNDEZ-GIMÉNEZ, M.E. y FILLAT, F. (2012 a): Pyrenean pastoralists' observations of environmental change: an exploratory study in los Valles Occidentales of Aragon. Pirineos, 167, 143-163.

FERNÁNDEZ-GIMÉNEZ, M.E. y FILLAT, F. (2012 b): Pyrenean pastoralists' ecological knowledge documentation and application to natural resource management and adaptation. Human Ecology, 40, 287-300.

FISHER, B., TURNER, R.K. y MORLING, P. (2009): Defining and classifying ecosystem services for decision making. Ecological Economics, 63, 616-626.

FOLEY, J.A., DEFRIES, R., ASNER, G.P., BARFORD, C., BONAN, G., CARPENTER, S.R., CHAPIN, F.S., COE, M.T., DAILY, G.C., GIBBS, H.K., HELKOWSKI, J.H., HOLLOWAY, T., HOWARD, E.A., KUCHARIK, C.J., MONFREDA, C., PATZ, J.A., PRETINCE, I.C., RAMANKUTTY, N. y SNYDER, P.K., (2005): Global consequences of land use. Science, 309, 570-574.

GALLART, F. y LLORENS, P. (2003): Catchment management under environmental change: Impact of land-cover change on water resources. Water International, 28, 334-340.

GALLART, F., LLORENS, P., LATRON, J. y REGÜÉS, D. (2002): Hydrological processes and their seasonal controls in a small Mediterranean mountain catchment in the Pyrenees. Hydrology and Earth System Sciences, 6 (3), 527-537.

GARCÍA-RUIZ, J.M. y LANA-RENAULT, N. (2011): Hydrological and erosive consequences of farmland abandonment in Europe, with special reference to the Mediterranean region - A review. Agriculture, Ecosystems and Environment, 140, 317-338.

GARCÍA-RUIZ, J.M., LASANTA, T. y SOBRÓN, I. (1988): Problemas de evolución geomorfológica en campos abandonados: el valle del Jubera (Sistema Ibérico): Zubía, 6, 99-114.

GARCÍA-RUIZ, J.M. y LASANTA-MARTÍNEZ, T. (1990): Land use changes in the Spanish Pyrenees. Mountain Research and Development, 10(3), 267-279. 
GARCÍA-RUIZ, J.M., LÓPEZ-MORENO, J.I., VICENTE-SERRANO, S., LASANTA, T. y BEGUERÍA, J.I. (2011): Mediterranean water resources in a global change scenario. Earth Science Reviews, 105 (3-4), 121-139.

GARCÍA-RUIZ, J.M., REGÜÉS, D., ALVERA, B., LANA-RENAULT, N., SERRANOMUELA, P., NADAL-ROMERO, E., LATRÓN, J., MARTÍ-BONO, C. y ARNÁEZ, J. (2008): Flood generation and sediment transport in experimental catchment affected by land use changes in the Central Pyrenees. Journal of Hydrology, 356 (1-2), 245-260.

GELLRICH, M., BAUR, P., KOCH, B. y ZIMMERMANN, N.E. (2007): Agricultural land abandonment and natural forest re-growth in the Swiss mountains: A spatially explicit economic analysis. Agriculture, Ecosytems and Environment, 118, 93-108.

GONZÁLEZ-MARTÍNEZ, S.C. y BRAVO, F. (2001): Density and population structure of the natural regeneration of Scots pine (Pinus sylvestris L.) in the High Ebro Basin (Northern Spain). Annales des Sciences Forestières, 58, 277-288.

HAIR, J.F., ANDERSON, R.E., TATHAM, R.L. y BLACH, W.C. (1999): Multivariate data analysis. Prentice Hall. Fifth edition. 799 pp.

KNAPP, C.N. y FERNÁNDEZ-GIMÉNEZ, M.E. (2009): Knowledge in practice: documenting rancher local knowledge in Northwest Colorado. Rangeland Ecology and Management, 62, 500-509.

KOMAC, B., KEFI, S., NUCHE, P., ESCÓS, J. y ALADOS, C.L. (2013): Modeling shrub encroachment in subalpine grasslands under different environmental and management scenarios. Journal of Environmental Management, 121, 160-169.

KOSMAS, C., DANALATOS, N.G. y GERONTIDIS, S.T. (2000): The effect of land parameters on vegetation performance and degree of erosion under Mediterranean conditions. Catena, 40, 3-17.

KOULOURI, M. y GIOURGA, CH. (2007): Land abandonment and slope gradient as key factors of soil erosion in Mediterranean terraced lands. Catena, 69, 274-281.

LASANTA, T. y ARNÁEZ, J. Eds. (2009): Gestión, usos del suelo y paisaje en Cameros (Sistema Ibérico, La Rioja). Universidad de La Rioja - Instituto de Estudios Riojanos: 367 pp., Logroño.

LASANTA, T. y ERREA, M.P. (2001): Despoblación y marginación en la Sierra Riojana. Instituto de Estudios Riojanos: 181 pp., Logroño.

LASANTA, T. y VICENTE-SERRANO, S.M. (2007): Cambios en la cubierta vegetal en el Pirineo Aragonés en los últimos 50 años. Pirineos, 162, 125-154.

LASANTA, T., ARNÁEZ, J., ERREA, M.P., ORTIGOSA, L. y RUIZ-FLAÑO, P. (2009): Mountain pastures, environmental degradation, and landscape remediation. The example of a Mediterranean Policy Initiative. Applied Geography, 29, 308-319.

LASANTA, T., ARNÁEZ, J., OSERIN, M. y ORTIGOSA, L. (2001): Marginal lands and erosion in terraced fields in the Mediterranean mountains: A case study in the Camero Viejo (Northwestern Iberian System, Spain). Mountain Research and Development, 21(1), 69-76.

LASANTA, T., ERREA, M.P., BOUZEBBOUDJA, M.R. y MEDRANO, L.M. (2013): Pastoreo y desbroce de matorrales en Cameros Viejo. Instituto de Estudios Riojanos, Colección Ciencias de la Tierra, no 30: 186 pp., Logroño. 
LASANTA, T., ERREA, M.P., VICENTE-SERRANO, S.M. y ARNÁEZ, J. (2011): La diversidad de la cubierta vegetal en campos abandonados del Leza y Jubera (Sistema Ibérico, La Rioja) a partir del Siose. Zubía. Monográfico, 23, 55-78.

LASANTA, T., NADAL-ROMERO, E., SERRANO-MUELA, P., VICENTE-SERRANO, S. y GARCÍA-RUIZ, J.M. (2010): Escorrentía y erosión tras el abandono de tierras de cultivo en montaña: resultados de la Estación Experimental «Valle de Aísa». Pirineos, 165: 115-133.

LASANTA, T., VICENTE-SERRANO, S. y CUADRAT, J.M. (2005): Mountain Mediterranean landscape evolution caused by the abandonment of traditional primary activities: A study of the Spanish Pyrenees. Applied Geography, 25, 47-65.

LAWRENCE, A. (2010): Introduction: learning from experiences of participatory biodiversity assessment. Taking Stock of Nature. Participatory Biodiversity Assessment for Policy, Planning and Practice /Ed. A. Lawrence), pp 1-29. Cambridge University Press, Cambridge.

LESSCHEN, J.P., CAMMERAAT, L.H. y NIEMAN, T. (2008): Erosion and terrace failure due to agricultural land abandonment in a semiarid environment. Earth Surface Processes and Landforms, 33(10), 1574-1584.

LLORENS, P. y DOMINGO, F. (2007): Rainfall partitioning by vegetation under Mediterranean conditions. A review of studies in Europe. Journal of Hydrology, 335 (1-2), $37-54$.

LÓPEZ-I-GELATS, F., TÀBARA, J.D. y BARTOLOMÉ, J. (2009): The rural in dispute: Discourses of rurality in the Pyrenees. Geoforum, 40: 602-612.

LÓPEZ-MORENO, J.I., VICENTE-SERRANO, S., MORÁN-TEJEDA, E., ZABALZA, J., LORENZO-LACRUZ, J., LÓPEZ-I-GELATS, F. y GARCÍA-RUIZ, J.M. (2011): Impact of climate evolution and land use changes on water yield in the Ebro basin. Hydrology Earth System Science, 15: 311-322.

MACDONALD, D., CRABTREE, J.R., WIEDINGER, G., DAX, T., STAMOU, N., FLEURY, P., GUTIÉRREZ-LAZPITA, J. y GIBON, A. (2000). Agricultural abandonment in mountain areas of Europe: environmental consequences and policy response. Journal Environmental Management, 59, 47-69.

MADRUGA-ANDREU, C., PLAIXATS, J., LÓPEZ-I-GELATS, F. y BARTOLOME, J. (2011). Medium-term success of revegetation methods for high-mountain grassland reclamation in the Montseny Biosphere Reserve (NE Spain). Plant Biosystems 145(3), 738-749.

MARCO, J., HERRERO, J., ESCUDERO, M.A., FERNÁNDEZ- ARBERAS, O., FERRERES, J., GARCÍA-SERRANO, A., GIMÉNEZ-ANAYA, A., LABARTA, J.L., MONRABAL, L. y PRADA, C. (2011): Veinte años de seguimiento poblacional de ungulados silvestres de Aragón. Pirineos, 166: 135-153.

MAREY-PÉREZ, M.F. y RODRÍGUEZ-VICENTE, V. (2009): Forest transition in Northern Spain: Local responses on large-scale programmes of field-afforestation. Land Use Policy, 26(1), 139-156.

MARTÍNEZ, J., VEGA-GARCÍA, C. y CHUVIECO, E. (2009): Human-caused wildfirerisk rating for prevention planning in Spain. Journal Environmental Management, 90: 12411252. 
MATAIX-SOLERA, J. y CERDA, A. (2009): Incendios forestales en España. Ecosistemas terrestres y suelos. En: A. Cerdà \& J. Mataix-Solera, Eds: Efectos de los incendios forestales sobre los suelos en España. El estado de la cuestión visto por los científicos españoles. Universidad de Valencia: 27-53, Valencia.

MOLINILLO, M., LASANTA, T. y GARCÍA-RUIZ, J.M. (1997). Managing mountainous degraded landscapes after farmland abandonment in the Central Spanish Pyrenees. Environmental Management, 21, 587-598.

MORÁN-ORDÓÑEZ A., SUÁREZ-SEOANE, S., CALVO, L. y DE LUIS, E. (2011). Using predictive models as a spatially explicit support tool managing cultural landscapes. Applied Geography, 31, 839-848.

MOREIRA, F., QUEIROZ, A.I. y ARONSON, J. (2006): Restoration principles applied to cultural landscapes. Journal for Nature Conservation, 14, 217-224.

MOREIRA, F., REGO, F.C. y FERREIRA, P.G. (2001): Temporal (1958-1995) pattern of change in a cultural landscape of northwestern Portugal: implications for five ocurrence. Landscape Ecology, 16, 587-598.

MOTTET, A., LADET, S., COQUE, N. y GIBÓN, A. (2006): Agricultural land-use change and its drivers in mountain landscapes: A case study in the Pyrenees. Agriculture, Ecosystems and Environment, 114, 296-310.

MULLER, B., LINSTADTER, A., FRANK, K., BOLLIG, M. y WISSEL, C. (2007): Learning from local knowledge: Modeling the pastoral-nomadic range management of the Himba, Namibia. Ecological Applications, 17, 1857-1875.

NADAL-ROMERO, E., LASANTA, T., GONZÁLEZ-HIDALGO, J.C., DE LUIS, J.M. y GARCÍA-RUIZ, J.M. (2012): The effect of intense rainstorm events on the suspended sediment response under various land uses: the Aísa Valley experimental station. Cuadernos de Investigación Geográfica, 38(1), 27-47.

NEARY, D.G., ICE, G.G. y JACKSON, C.R. (2009): Linkages between forest soils and water quality and quantity. Forest Ecology and Management, 258 (10): 2269-2281.

O'ROURKE, E. (2006): Biodiversity and land use change on the cause Mejan, France. Biodiversity Conservation, 15, 2611-2626.

OHL, C., JOHST, K., MEYERHOFF, J., BECKENKAMP, M., GRÜSGEN, V. y DRESCHSLER, M. (2010): Long-term socio-ecological research (LTSER) for biodiversiyy protection -a complex system approach for the study of dynamic human-nature interactions. Ecological Complexity, 7, 170-178.

ORTIGOSA, L.M., GARCÍA-RUIZ, J.M. y GIL, E. (1990): Land reclamation by reforestation in the Central Pyrenees. Mountain Research and Development, 10(3), 281-288.

OSTROM, E. (2009): General framework for analyzing sustainability of social-ecological systems. Science, 325, 419-422.

PALAHI, M., MAVSAR, R., GRACIA, C. y BIROT, Y. (2008): Mediterranean forest under focus. International Forestry Review, 10, 676-688.

PAUSAS, J.G. y KEELEY, J.E. (2009): A burning story: the role of fire in the history of life. Bioscience, 59(7), 593-601.

PÉREZ, J.M., GRANADOS, J.E., SORIGUER, R., FANDOS, P., MÁRQUEZ, F.J. y CRAMPE, J.P. (2002): Distribution, status and conservation problems of the Spanish ibes Capra pyrenaica (Mammalia: Artiodactyla). Mammalia Revue, 32, 26-39. 
PUEYO, Y. y BEGUERÍA, S. (2007): Modelling the rate of secondary succession after farmland abandonment in a Mediterranean mountain area. Landscape and Urban Planning, 83: $245-254$.

ROTURIER, S. y ROUE, M. (2009): Of forest, snow and lichen: Sami reindeer herders' knowledge of winter pastures in Northern Sweden. Forest Ecology and Management, 258, 1969-1967.

ROURA-PASCUAL, N., PONS, P., ETIENNE, M. y LAMBERT, B. (2005): Transformation of a rural landscape in the Eastern Pyrenees between 1953 and 2000. Mountain Research and Development, 25, 252-261.

RUIZ-MIRAZO, J. y ROBLES, A.B. (2012): Impact of targeted sheep grazing on herbage and holm oak saplings in a silvopastoral wildfire prevention system in south-eastern Spain. Agroforestry System, 86, 477-491.

SANTANA, VM., BAEZA, MJ., MARRS, RH. y VALLEJO VR. (2010). Old-field secondary succession in SE Spain: can fire divert it? Plant Ecology, 211, 337-349.

SAYADI, S., GONZÁLEZ-ROA, MC. y CALATRAVA REQUENA, J. (2009). Public preferences for landscape features: The case of agricultural landscape in mountainous Mediterranean areas. Land Use Policy 26, 334-344.

SCARASCIA-MUGNOZZA, G., OSWALD, H., PIUSSI, P. y RADOGLOU, K. (2000): Forest of the Mediterranean region: gaps in knowledge and research needs. Forest Ecology and Management, 132, 97-109.

SCHMITZ, M.F., DE ARANZABAL, I. y PINEDA, F.D. (2007): Spatial analysis of visitor preferences in the outdoor recreational niche of Mediterranean cultural landscapes. Environmental Conservation, 34, 300-312.

SCHMITZ, M.F., DE ARANZABAL, I., AGUILERA, P., RESCIA, A.J. y PINEDA, F.D. (2003): Relationship between landscape typology and socioeconomic structure: Scenarios of change in Spanish cultural landscapes. Ecological Modelling, 168, 349-356.

SERRA, P., PONS, X. y SAURI, D. (2008). Land-cover and land-use change in a Mediterranean landscape: a spatial analysis of driving forces integrating biophysical and human factors. Applied Geography, 28, 189-209.

SERRANO-MUELA, P., LANA-RENAULT, N., NADAL-ROMERO, E., REGÜÉS, D., LATRON, J., MARTÍ-BONO, C. y GARCÍA-RUIZ, J.M. (2008): Forest and their hydrological influence in mediterranean mountains. The case of the Central Spanish Pyrenees. Mountain Research and Development, 23 (3-4), 279-285.

SFERI, E., VICENTE-SERRANO, S., GONZÁLEZ-HIDALGO, J.C. y LASANTA, T. (2005): ¿Hacia dónde evolucionaría la composición del paisaje si la actividad humana fuera mínima? Una aproximación a los cambios probables del paisaje en el Pirineo central español. Estudios Geográficos, 259, 667-687.

SHEIL, D. y LAWRENCE, A. (2004): Tropical biologist, local people and conservation: new opportunities for collaboration. Trends in Ecology \& Evolution, 19, 634-638.

SLUITER, R. y DE JONGS, S.M. (2007): Spatial patterns of Mediterranean land abandonment and related land cover transitions. Landscape Ecology, 22, 559-576.

SOLIVA, R. (2007): Landscape stories: Using ideal type narratives as a heuristic device in rural studies. Journal of Rural Studies, 23, 62-74. 
STRIJKER, D. (2005): Marginal lands in Europe - causes of decline. Basic and Applied Ecology, 6, 99-106.

TAILLEFUMIER, F. y PIÉGAY, H. (2003). Contemporary land use changes in Prealpine Mediterranean mountains: a multivariate GIS-Based approach applied to two municipalities in the Southern French Prealps. Catena, 51, 267-296.

TASSER, E., WALDE, J., TAPPEINER, U., TEUTSCH, A. y NOGGLER, W. (2007): Landuse changes and natural reforestation in the Eastern Central Alps. Agriculture, Ecosystems and Environment, 118, 115-129.

UBALDE, J.M., RIUS, J., y POCH, R.M. (1999): Monitorización de los cambios de uso del suelo en la cabecera de cuenca de la ribera Salada mediante fotografía aérea y S.I.G. (El Solsonés, Lleida, España). Pirineos, 153-154, 101-122.

VAN AUKEN, O.W. (2009): Causes and consequences of woody encroachment into western North American grasslands. Journal of Environmental Management, 90, 2931-2942.

VICENTE-SERRANO, S.M., LASANTA, T. y ROMO, A. (2005). Analysis of the spatial and temporal evolution of vegetation cover in the Spanish Central Pyrenees. The role of Human Management. Environmental Management, 34(6), 802-818.

VILA-SUBIRÓS, J., RIBAS-PALOM, A., VARGA-LINDE, D. y LLAUSÀS-PASCUAL, A. (2009): Medio siglo de cambios paisajísticos en la montaña mediterránea. Percepción y valoración social del paisaje en la alta Garrotxa (Girona). Pirineos, 164, 69-92.

VINUESA ANGULO, J. (2005): De la población de hecho a la población vinculada. Cuadernos Geográficos, 36, 79-90.

WALLACE, K.J. (2007): Classifications of ecosystem services: problems and solutions. Biological Conservation, 139, 235-246. 